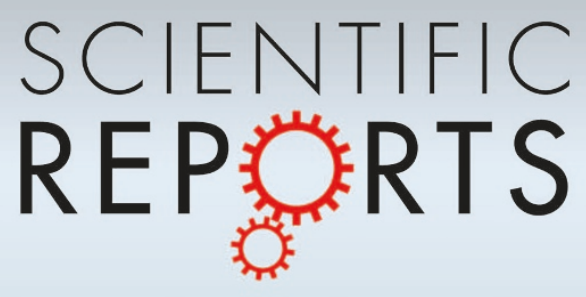

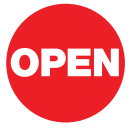

SUBJECT AREAS:

ELECTRONIC PROPERTIES AND MATERIALS

SUPERCONDUCTING PROPERTIES AND MATERIALS

SPECTROSCOPY

ELECTRONIC STRUCTURE

Received

18 January 2013

Accepted

24 April 2013

Published

13 May 2013

Correspondence and requests for materials should be addressed to D.M. (dmeyers@uark. edu) or T.S.-D. (t. sahadasgupta@gmail.

com)

\section{Zhang-Rice physics and anomalous copper states in A-site ordered perovskites}

\author{
D. Meyers' ', Swarnakamal Mukherjee'2, J.-G. Cheng'3,4,5, S. Middey' , J.-S. Zhou ${ }^{3}$, J. B. Goodenough ${ }^{3}$, \\ B. A. Gray', J. W. Freeland ${ }^{6}$, T. Saha-Dasgupta ${ }^{2} \&$ J. Chakhalian ${ }^{1,7}$
}

'Department of Physics, University of Arkansas, Fayetteville, AR 72701, ${ }^{2}$ Department of Condensed Matter Physics and Materials Science, S. N. Bose National Centre for Basic Sciences, Kolkata 700098, India, ${ }^{3}$ Texas Materials Institute, ETC 9. 102, University of Texas at Austin, Austin, Texas 78712, ${ }^{4}$ Institute for Solid State Physics, University of Tokyo, 5-1-5 Kashiwanoha, Kashiwa, Chiba Japan 277-8581, ${ }^{5}$ Beijing National Laboratory for Condensed Matter Physics, and Institute of Physics, Chinese Academy of Sciences, Beijing 100190, China, ${ }^{6}$ Advanced Photon Source, Argonne National Laboratory, Argonne, Illinois 60439, USA, ${ }^{7}$ Division of Physics and Applied Physics, School of Physical and Mathematical Sciences, Nanyang Technological University, Singapore 637371 , Singapore.

In low dimensional cuprates several interesting phenomena, including high $\mathrm{T}_{c}$ superconductivity, are deeply connected to electron correlations on $\mathrm{Cu}$ and the presence of the Zhang-Rice (ZR) singlet state. Here, we report on direct spectroscopic observation of the ZR state responsible for the low-energy physical properties in two isostructural A-site ordered cuprate perovskites, $\mathrm{CaCu}_{3} \mathrm{Co}_{4} \mathrm{O}_{12}$ and $\mathrm{CaCu}_{3} \mathrm{Cr}_{4} \mathrm{O}_{12}$ as revealed by resonant soft $\mathrm{x}$-ray absorption spectroscopy on the $\mathrm{Cu} \mathrm{L}_{3,2^{-}}$and $\mathrm{O}$ K-edges. These measurements reveal the signature of $\mathrm{Cu}$ in the high-energy $3+\left(3 \mathrm{~d}^{8}\right)$, the typical $2+\left(3 \mathrm{~d}^{9}\right)$, as well as features of the $\mathrm{ZR}$ singlet state (i.e., $3 \mathrm{~d}^{9} \underline{L}, \underline{L}$ denotes an oxygen hole). First principles GGA $+U$ calculations affirm that the $\mathrm{B}$-site cation controls the degree of $\mathrm{Cu}-\mathrm{O}$ hybridization and, thus, the $\mathrm{Cu}$ valency. These findings introduce another avenue for the study and manipulation of cuprates, bypassing the complexities inherent to conventional chemical doping (i.e. disorder) that hinder the relevant physics.

 he many-body ZR singlet state ${ }^{1-9}$, a doped hole on the oxygen site coupled antiferromagnetically with a hole on the copper site, can be manipulated with conventional solid-state chemistry methods by partial removal of oxygen or by non-isovalent cation exchange $\left(e . g . \mathrm{La}^{3+} \rightarrow \mathrm{Sr}^{2+}\right)$. Such chemical routes of manipulation make the the doped cuprates prone to chemical disorder causing strong structural distortions or even changes in crystal symmetry, which in turn may severely alter the properties associated with $\mathrm{Cu} d$-electron derived electronic and magnetic structures. En route to the goal of realizing the ZR state without chemical disorder or lattice distortion, while the majority of the $\mathrm{Cu}$ oxide compounds have the $\mathrm{Cu}$ ions in the $\mathrm{B}$-site of the perovskite $\mathrm{ABO}_{3}$ structure, the A-site ordered perovskites with chemical formula $\left(\mathrm{ACu}_{3}\right) \mathrm{B}_{4} \mathrm{O}_{12}$ are intriguing candidates for investigation due to the unique $\mathrm{Cu} \mathrm{A}$-site arrangement (see Fig. 1(a)). In this structure, a surprisingly rich set of interesting physics phenomena ${ }^{10-16}$ have been demonstrated by replacement of the B-site $3 d$ transition metal ion via experiment and theory including colossal dielectric constant ${ }^{17,18}$, ferrimagnetism ${ }^{19}$, charge, orbital, and spin ordering ${ }^{20}$, non-fermi liquid behavior ${ }^{21}$, enhanced electronic correlations ${ }^{22}$ and possibly the Zhang-Rice state $^{23}$. In addition, previous reports hinted at the presence of a mixed-valency on Cu for $\mathrm{B}=\mathrm{Cr}$ and $\mathrm{Co}^{23,24}$. Despite the large variation in the B-site ions, all the members of this family belong to the same space group Im-3 built upon two sub-lattices of octahedral $\mathrm{BO}_{6}$ units and the planar $\mathrm{CuO}_{4}$ units (see Fig. 1(b)-(c). As seen in Fig. 1(a and $\mathrm{d}$ ), the two structural units are connected via apical oxygens to form a $\mathrm{BO}_{6}-\mathrm{CuO}_{4}-\mathrm{BO}_{6}$ cluster. In this configuration, the effect of the B-site cation has been shown to be two-fold: first, its valence state controls the nominal valence state of the $\mathrm{A}^{\prime}$-site $\mathrm{Cu}$ ion and second, most importantly, its tendency for covalent mixing alters the charge distribution between $\mathrm{Cu} d$ - and $\mathrm{O}$ p-states ${ }^{11,15}$. The structural similarity of the local ionic environment of $\mathrm{Cu}$ in these ordered perovskites with that of high $\mathrm{T}_{c}$ cuprates, and, in close analogy to chemical doping, the above-mentioned B-site's potential to engineer the electronic structure prompts one to use the A-site ordered perovskites to gain unique insight into the development of the cuprate electronic structure. Construction of such manipulated valencies suggests the ability to tune the formation of ligand holes on oxygen and possibly to achieve the formation of the Zhang-Rice singlet state - an important ingredient for high temperature superconductivity. 


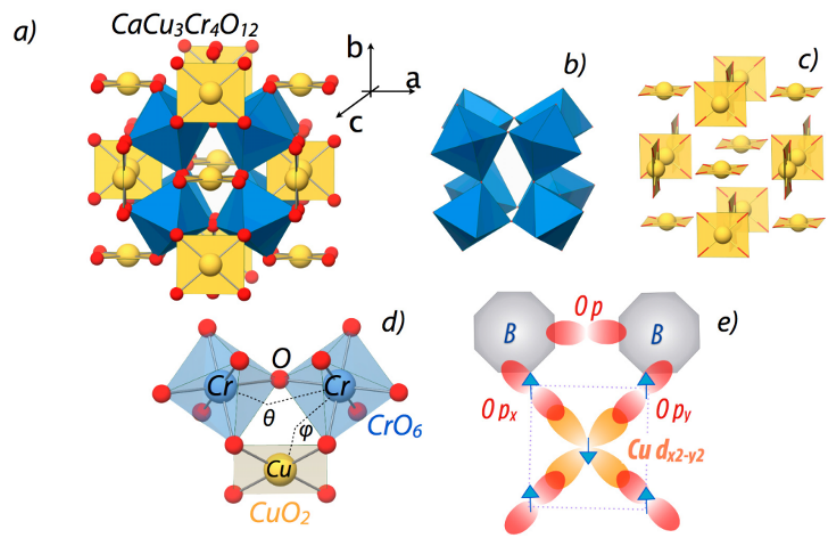

Figure $1 \mid$ Crystal structure of A-site ordered perovskites. (a) The entire crystal structure of $\mathrm{CaCu}_{3} \mathrm{Cr}_{4} \mathrm{O}_{12}$. (b) $\mathrm{CrO}_{6}$ octahedral sub-lattice. (c) $\mathrm{CuO}_{4}$ distorted square planar sub-lattice. (d) $\mathrm{Cr}-\mathrm{Cr}$ and $\mathrm{Cr}-\mathrm{Cu}$ exchange pathways and (e) a sketch of B-site electronic states coupled to the ZR singlet state on $\mathrm{Cu}$.

\section{Results}

Resonant X-ray absorption spectroscopy (XAS) in the soft X-ray regime is an ideal tool, extensively used in the past, to investigate the $\mathrm{Cu}$ and $\mathrm{O}$ electronic structure and the effects of electron-electron correlations in high $\mathrm{T}_{c}$ cuprates $^{2,6-8,25-27}$. Results of our soft XAS measurements on the $\mathrm{Cu} \mathrm{L}_{3,2^{-}}$and $\mathrm{O} \mathrm{K}$-edge are shown in Fig. 2 for the two A-site ordered perovskites $\mathrm{CaCu}_{3} \mathrm{Co}_{4} \mathrm{O}_{12}(\mathrm{CCCoO})$ and $\mathrm{CaCu}_{3} \mathrm{Cr}_{4} \mathrm{O}_{12}$ (CCCrO) along with that of the optimally doped superconducting $\mathrm{YBa}_{2} \mathrm{Cu}_{3} \mathrm{O}_{7-\delta}(\mathrm{YBCO})$ and metallic $\mathrm{LaCuO}_{3}$ (LCO) perovskite with a unique formal $\mathrm{Cu}^{3+}\left(3 \mathrm{~d}^{8}\right)$ oxidation state. From the absorption line shape in Fig. 2, it is readily apparent that in $\mathrm{CCCoO}$ and $\mathrm{CCCrO}$ a mixed valency of $\mathrm{Cu}$ is present; we will discuss the CCCrO compound first. The sharp peak $\sim 930 \mathrm{eV}$ corresponds to the transition from the $\mathrm{d}^{9}$ ground state, featuring a single hole in the $\mathrm{Cu} \mathrm{e} g$ band, to the $\mathrm{cd}^{10}$ (here $\underline{c}$ denotes a core hole) excited state. The shoulder seen at $9 \overline{3} 1.5 \mathrm{eV}$, a signature of the ZR singlet state, is a transition from the ground state $\mathrm{d}^{9} \mathrm{~L}$ to the $\mathrm{cd}^{10} \mathrm{~L}$ excited state $\mathrm{e}^{2,5,7,26,27}$. The key difference between these two transitions being that while the $\mathrm{Cu}$ still maintains a nearly $2+\left(\right.$ or $\left.\mathrm{d}^{9}\right)$ valency, a ligand hole is distributed over the neighboring oxygens as illustrated in Fig. 1(e). The interaction between this ligand hole and the core hole created by the photon absorption raises the energy required to promote the core electron to the unoccupied state in the $\mathrm{e}_{g}$ band resulting in the observed high-energy state marked by red arrows. Furthermore, to corroborate this, we show the absorption data on LCO with the formal $\mathrm{Cu}$ charge state of $3+$. Indeed, in-line with our previous statement, the main feature of the LCO XAS around the $\mathrm{L}_{3}$-edge at $\sim 931.5 \mathrm{eV}$ is the transition from the $\mathrm{d}^{9} \mathrm{~L}$ state, thus making the $\mathrm{ZR}$ state the dominant contribution to the ground state in good agreement with previous reports ${ }^{28,29}$. The aforementioned doped ligand holes are the charge carriers essential to the physics of high $\mathrm{T}_{c}$ cuprates; for instance, in hole doped cuprates the superconducting transition temperature is strongly dependent on the amount of doping present $^{2,30,31}$. To clarify the connection between these compounds and the high-temperature superconducting cuprates, XAS data was taken for an optimally doped YBCO sample. As seen in Fig. 2, the $\mathrm{Cu}$ L-edge spectrum of CCCrO shows remarkable resemblance to YBCO, namely the dominant $\mathrm{d}^{9}$ initial states followed by the higher energy ZR state shoulder. This result highlights the capacity of the Asite ordered cuprate perovskites to mimic the effects of chemical doping without provoking unwanted lattice and stoichiometry deviations inherent to conventional chemical doping. In addition, the multiplet split peak at $\sim 940 \mathrm{eV}$ corresponds to a transition from

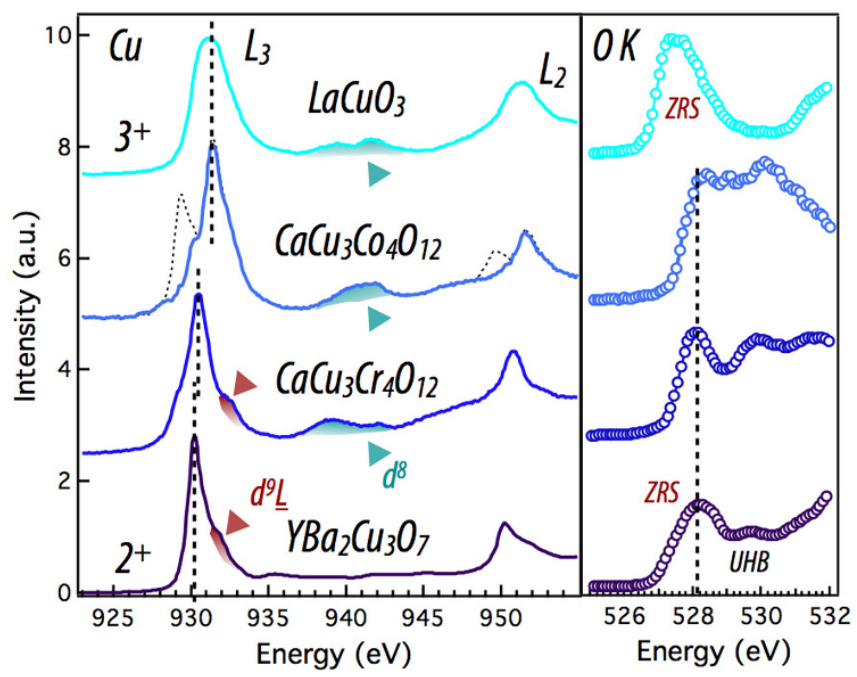

Figure $2 \mid$ XAS measurements on Cu L- and O K-edges. Left panel displays the $\mathrm{Cu}$ L-edge for all samples with the Zhang-Rice and $\mathrm{d}^{8}$ ground states highlighted. LCO and YBCO are shown as references for $\mathrm{Cu} 3+$ and $\mathrm{Cu} 2+$ respectively, highlighting the change in valence for $\mathrm{Cu}$ with $\mathrm{B}$-site change ${ }^{36,37}$. Right panel shows the corresponding $\mathrm{O}$ K-edge spectra displaying the Zhang-Rice ligand hole state. In LCO, the higher energy peak around $\sim 940 \mathrm{eV}$ is due to the presence of the unusual $\mathrm{Cu} \mathrm{d}^{8}$ state. In $\mathrm{CCCoO}$, a previously reported impurity peak around $929 \mathrm{eV}^{32}$ is subtracted from both the $\mathrm{L}_{3}$ and $\mathrm{L}_{2}$ edges by fitting to a Voigt function. This peak corresponds to the $\mathrm{d}^{9}$ peak observed on the other samples, however it has a very small spectral weight ( $\sim 1 / 50$ ratio between $\mathrm{d}^{9}$ and $\mathrm{d}^{9} \mathrm{~L}$ states).

the metastable $3 \mathrm{~d}^{8}$ state to the $\mathrm{cd}^{9}$ excited state, indicating the presence of the ionically $\mathrm{Cu}^{3+}$ state entirely absent in YBCO.

Next, we turn our attention to the $\mathrm{CCCoO}$ electronic structure. As clearly seen in Fig. 2, CCCoO shows a dominant white absorption line analogous to CCCrO, but, surprisingly, it is shifted $\sim 1 \mathrm{eV}$ away from the $\mathrm{d}^{9}$ white line of $\mathrm{CCCrO}$ and YBCO. When compared with the formally $\mathrm{Cu}^{3+}$ compound LCO (shown immediately above it in Fig. 2) it becomes readily apparent that the $\mathrm{Cu}$ ground state is predominantly of $\mathrm{d}^{9} \mathrm{~L}$ character, implying a dominant $\mathrm{Cu}^{3+}$ formal valence as in LCO. To quantify the prevalence of the $\mathrm{d}^{9} \mathrm{~L}$ state, a three peak fit is used for the $\mathrm{L}_{3}$ data, which are centered at $929.3 \mathrm{eV}$ (impurity) $)^{32}, 930 \mathrm{eV}\left(\mathrm{d}^{9}\right)$, and $931.4 \mathrm{eV}\left(\mathrm{d}^{9} \mathrm{~L}\right)$ which gave a very small spectral weight ratio of $.0203\left(\frac{d^{9}}{d^{9} \underline{L}}\right)$. The impurity peak was subtracted from the original data (dotted line) for clarity. Further demonstrating the $3+$ formal valence, the multiplet split peak at $\sim 940 \mathrm{eV}$ provides strong evidence for the admixture of the $\mathrm{d}^{8}$ state. When considered alongside CCCrO, CCCoO also contains all three $\mathrm{Cu}$ states, but with obviously significantly different weights, further testifying to the ability of the B-site to effectively hole dope the $\mathrm{Cu}$ sites.

Because of the large degree of charge transfer between oxygen and copper and its importance to the ZR state, studies of the O K-edge can lend supporting information about the electronic structure of $\mathrm{Cu}$. As seen in Fig. 2(b), O K-edge data in all samples show the oxygen specific signature of the $3 \mathrm{~d}^{9} \mathrm{~L}$ state, a necessary ingredient of the ZR state, which is particularly evident from the comparison to the well studied case of YBCO, where the ligand hole on oxygen appears as a large, doping dependent pre-peak around $528 \mathrm{eV}^{6-8,25}$. This observation of the pre-peak intensity indicates that the ZR state is the first ionization state at the Fermi energy. This further evidences our conclusion on the presence of the ZR state inferred from the $\mathrm{Cu}$ L-edge analysis.

Although our prime focus is on the electronic structure of $\mathrm{Cu}$ and $\mathrm{O}$, Co and $\mathrm{Cr} \mathrm{L}_{3,2}$-edge absorption can serve to independently 

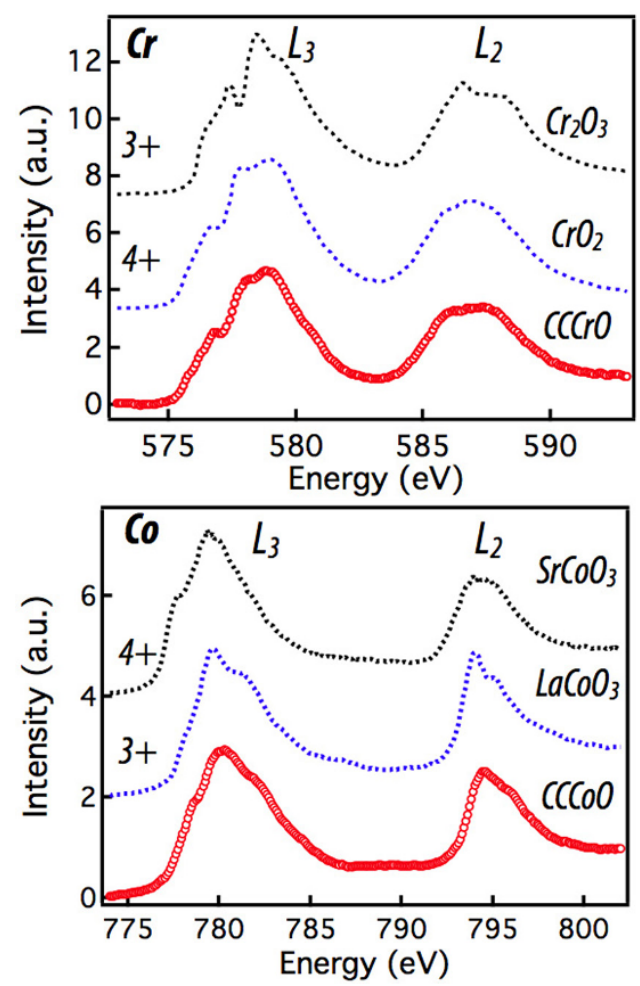

Figure 3 XAS measurements on the Cr and Co L-edges. (a) The Cr Ledge along with $3+$ and $4+$ references from ${ }^{33}$, the CCCrO and the $4+$ reference samples show strong similarities. (b) The Co L-edge with valence references from ${ }^{34}$ showing a similar, but not identical line shape. No standards were available meaning any energy shift between the samples could not be analyzed, all spectra were shifted to $\sim$ align the white lines.

validate the $\mathrm{Cu}$ oxidation state. With this goal in mind, we performed L-edge XAS measurements on Co and $\mathrm{Cr}$ and compared this to the previous results on reference $\mathrm{Cr}$ and Co compounds. As shown in Fig. 3(a), a direct comparison to the $\mathrm{Cr}^{3+}$ and $\mathrm{Cr}^{4+}$ absorption confirms that in CCCrO the $\mathrm{Cr}$ valency is $4+$ or very close to $\mathrm{it}^{33}$. This verifies the conclusion that, in this compound, if $\mathrm{Cr}$ is $4+$ then $\mathrm{Cu}$ must be $\sim 2+$ (assuming $\mathrm{O}^{2-}$ and $\mathrm{Ca}^{2+}$ ). Any deviation of $\mathrm{Cr}$ valency towards $3+$ must then be compensated by raising the $\mathrm{Cu}$ charge state, thus $\mathrm{Cu}$ must be $2+$ or very close to it, consistent with the $\mathrm{Cu}$ L-edge results. The Co L-edge data shown in Fig. 3(b) bear strong similarities with previous studies of $\mathrm{LaCoO}_{3}$, which consists of ionically bound $\mathrm{Co}^{3+34}$. Specifically, strong, high energy multiplet shoulders on the $\mathrm{L}_{3}$ and $\mathrm{L}_{2}$ white lines are present in both CCCoO and $\mathrm{LaCoO}_{3}$. In variance to this, $\mathrm{Co}^{4+}$ shows a smaller shoulder shifted to even higher energy. While the high energy side of the CCCoO Co L-edge is consistent with $\mathrm{Co}^{3+}$, the low energy multiplet peak does not match either $3+$ or $4+$, implying CCCoO is neither ionically pure $3+$ or $4+$. Based upon the $\mathrm{Cu}$ L-edge conclusion of the $\mathrm{Cu}^{3+}$ charge state, we can independently assess the Co valency to be $\sim 3.25+$. In this context, the Co L-edge data is compatible with the $\mathrm{Cu}$ L-edge results and implies the presence of a mixed $3+/ 4+\mathrm{Co}$ valency, although it cannot confirm the exact $3.25+$ valence state needed to conserve charge. Lending further support to this conclusion, Co L edge data in total electron yield (TEY) mode is shown in the Supplemental Figure S1.

To gain further understanding of the B-site influence on the unusual electronic states on $\mathrm{Cu}$, we performed first-principles spin-polarized DFT calculations within the GGA $+U$ approximation. Fig. 4 summarizes the first-principles results for $\mathrm{CCCoO}$ and $\mathrm{CCCrO}$, respectively. Note, although no long-range magnetic ordering is reported in these compounds, spin-polarized calculations are
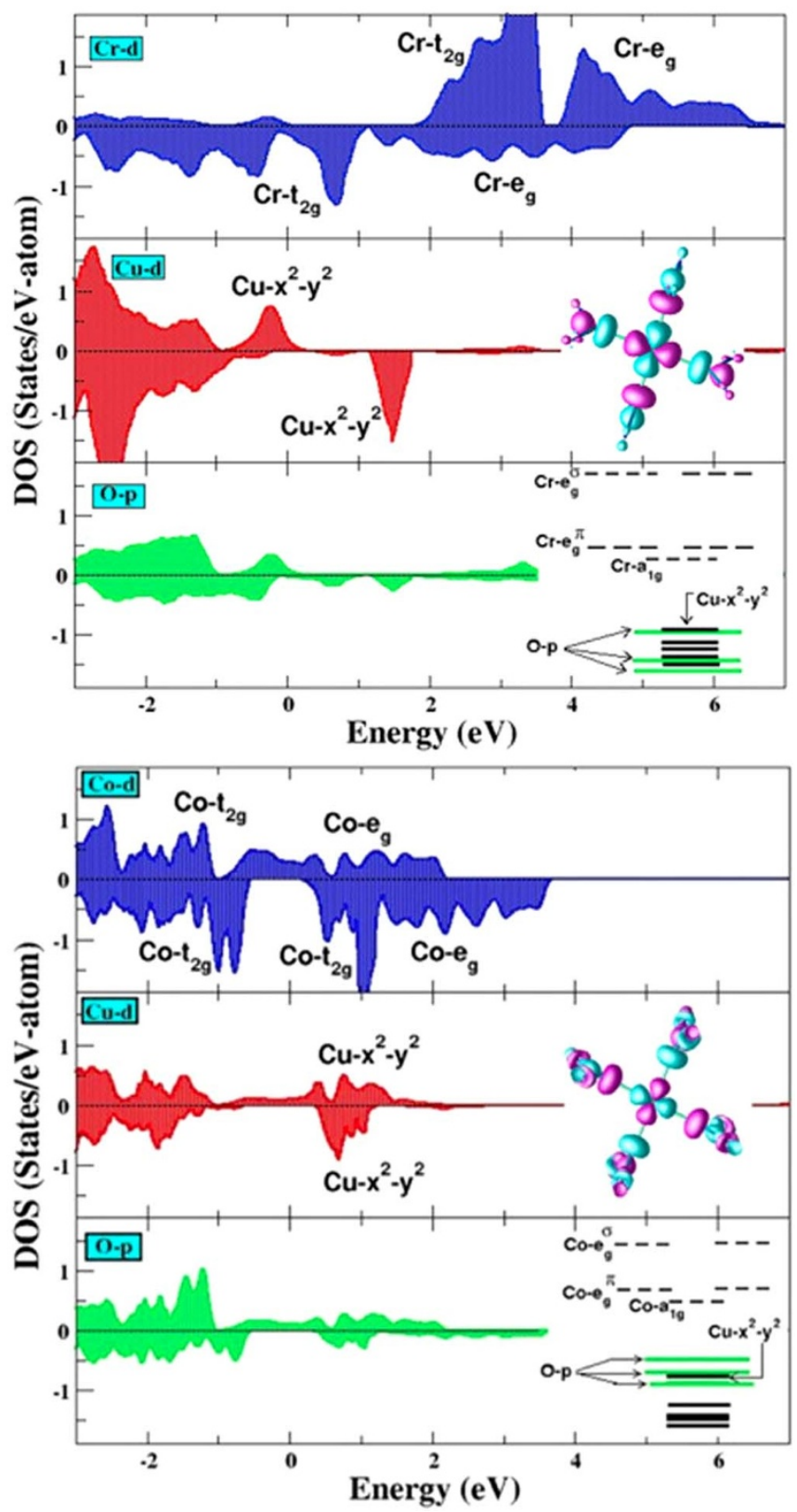

Figure $4 \mid$ The GGA + Udensity of states, projected onto Co d (Cr d), Cu $\mathrm{d}$ and $\mathrm{O} \mathrm{p}$ states for the $\mathrm{A}$-site ordered perovskites. (a) $\mathrm{CaCu}_{3} \mathrm{Co}_{4} \mathrm{O}_{12}$ ( (b) $\left.\mathrm{CaCu}_{3} \mathrm{Cr}_{4} \mathrm{O}_{12}\right)$ compound. The positive and negative values in $\mathrm{y}$-axis correspond to density of states inup and down spin channels, respectively. The zero of the energy is set at $\mathrm{E}_{F}$. The features due to dominant contributions of various degrees of freedom have been marked. The inset in the middle panel shows the plot of the effective $\mathrm{Cu} x^{2}-y^{2}$ Wannier function, calculated by NMTO-downfolding. Shown are the isosurface with lobes of opposite signs are colored differently. The inset in the bottom panel shows the relative positions of $\mathrm{Co} \mathrm{d}, \mathrm{Cu} \mathrm{d}$ and $\mathrm{O}$ p energy levels.

essential for proper assignment of both charge and spin states of various constituents ${ }^{15}$. The three panel Fig. 4(a) and (b) show an inspection of the calculated density of states (DOS) projected onto B$\mathrm{d}(\mathrm{B}=\mathrm{Co} / \mathrm{Cr}), \mathrm{Cu}-\mathrm{d}$ and $\mathrm{O}-\mathrm{p}$ states respectively. A direct inspection of the states in the vicinity to the Fermi energy, $\mathrm{E}_{\mathrm{F}}$ demonstrates the existence of strong mixing between $\mathrm{B}$ and $\mathrm{Cu}$ d-states and $\mathrm{O}$ p-states. This strong covalency effect makes both CCCoO and CCCrO systems metallic, in agreement with experimental observations. In addition, as shown in the inset of Fig. 4(a)-(b), the octahedral crystal field 
of the oxygen atoms around the B ion splits the B- $\mathrm{t}_{2 g}$ states from the $\mathrm{B}-\mathrm{e}_{g}\left(\mathrm{e}_{g}^{\sigma}\right)$ states; the trigonal distortion present in the $\mathrm{BO}_{6}$ octahedra breaks the $t_{2 g}$ levels further into doubly degenerate, $\mathrm{e}_{g}^{\pi}$ and singly degenerate $\mathrm{a}_{1 g}$ states. The distorted square planar environment of oxygen atoms surrounding the $\mathrm{Cu}$ ion, on the other hand, lifts the 5 fold degeneracy of $\mathrm{Cu}$ d-levels, with large energy separation of $\mathrm{Cu}$ $\mathrm{d}_{x^{2}-y^{2}}$ state from the rest. Here we note that the nominal $\mathrm{d}^{8}$ (i.e. $\mathrm{Cu}^{3+}$ ) would imply empty $\mathrm{Cu}: \mathrm{d}_{x^{2}-y^{2}}$ states in both spin up and spin down channels, and for $\mathrm{d}^{9} \mathrm{Cu}: \mathrm{d}_{x^{2}-y^{2}}$ states would be occupied in one of the spin channels and be empty in the other spin channel. From the DOS plots shown in Fig. 4(a) and (b), we find that $\mathrm{Cu}$ : $\mathrm{d}_{x^{2}-y^{2}}$ states are empty in the down spin channel, while in the up spin channel it is indeed close to being empty for CCCoO, but mostly occupied for CCCrO. We note that DFT calculation deals with single configuration nature of the wavefunction and therefore, delivers the state with most dominant weight. Interestingly enough, the dominance of $\mathrm{Cu} 3+$ state in $\mathrm{CCCoO}$ and $\mathrm{Cu} 2+$ state in CCCrO is evident even at the level of single configuration theory. This result lends strong theoretical support for the conclusion inferred from our XAS measurements that the $\mathrm{Cu}$ state is predominantly in the $3+$ state for $\mathrm{CCCoO}$ and closer to $2+$ for CCCrO.

Next, we discuss the calculated magnetic states in connection to $\mathrm{Cu}$. First, we note that occupancy of the square planar $\mathrm{Cu}$ d-states according to the formal valence of $\mathrm{d}^{8}$ would give rise to a paired electronic configuration with zero magnetic moment at $\mathrm{Cu}$ sites, while that of formal valence of $\mathrm{d}^{9}$ would give rise to one unpaired electron with magnetic moment of $1 \mu_{B}$ in the fully spin polarized limit. In comparison, the calculation yields magnetic moments on $\mathrm{Cu}$ site $0.07 \mu_{B}$ for $\mathrm{CCCoO}$ and $0.50 \mu_{B}$ for CCCrO, thus lending additional validation of our experimental conclusion on the exotic $3+$ state for $\mathrm{CCCoO}$ and closer to $2+$ valency in CCCrO. Moreover, from the simple ionic charge count, the nominal $3+$ and $2+$ valences of $\mathrm{Cu}$ (assuming $\mathrm{Ca}^{2+}$ and $\mathrm{O}^{2-}$ ), would set the formal valences of the B-site ion to $3.25+$ and $4+$ respectively, and result in d-electron occupancies of 5.75 for $\mathrm{Co}$ and 2 for $\mathrm{Cr}$. The computed magnetic moment at the $\mathrm{Cr}$ site is $2.2 \mu_{B}$, in good agreement with the nearly $2+$ valence of $\mathrm{Cu}$ and $4+$ valence of $\mathrm{Cr}$ in CCCrO. The d-occupancy of 5.75 on Co can lead to either low or high spin magnetic state; our computed Co magnetic moment of $\sim 1.7 \mu_{B}$, however, is in conformity with the intermediate rather than low- or high-spin state.

By examining the energy level positions, as calculated in DFT, shown in the insets in the bottom panels of Figs. 4(a-b), we find that $\mathrm{Cu} \mathrm{d}_{x^{2}-y^{2}}$ energy level is nearly degenerate with $\mathrm{O}-p$ states, leading to a situation very similar to that in high $\mathrm{T}_{c}$ cuprates. This electronic configuration naturally supports strong mixing of $\mathrm{Cu}$ $\mathrm{d}_{x^{2}-y^{2}}$ and $\mathrm{O} \mathrm{p}_{x}$ and $\mathrm{p}_{y}$ states by forming a strong $\mathrm{pd} \sigma$ antibonding combination, which may further bind to d-states at the B site as shown in Fig. 1(e). The formation of such a mixed state is also evident in the Wannier function plot of an effective $\mathrm{Cu} x^{2}-$ $y^{2}$ Wannier function obtained by keeping only $\mathrm{Cu} \mathrm{d}_{x^{2}-y^{2}}$ state active and downfolding or integrating out all the other degrees of freedom (see inset of the middle panel of Fig. 4). We notice that for the Co compound mixing of $\mathrm{Cu}-\mathrm{O}$ hybridized states with $\mathrm{B}$ site d-states is much stronger compared to that of $\mathrm{Cr}$ compound, which is driven by the strong hybridization of empty $\mathrm{Cu}_{x^{2}-y^{2}}$ orbital of $\mathrm{Cu} 3+$ state with empty $\mathrm{Co}, \mathrm{e}_{g}$ orbitals. In CCCrO, this leads to vanishing contribution of the B-site states for the selected iso-surface value of the Wannier plot, showing the typical $\mathrm{Cu}-\mathrm{O}$ antibonding wavefunction, making the situation more like high $\mathrm{T}_{c}$ cuprates with dominant $\mathrm{Cu} 2+$ state. The strong mixing between $\mathrm{Cu}_{x^{2}-y^{2}}$ and $\mathrm{O} \mathrm{p}$ states, which is a prerequisite for formation of the ZR state - observed as the feature around $931 \mathrm{eV}$ in $\mathrm{Cu} \mathrm{L}$-edge spectra, is further corroborated by the presence of a $0.2 \mu_{B}$ fraction of the magnetic moment on the $\mathrm{O}$ site and $0.5 \mu_{B}$ on $\mathrm{Cu}$. We thus conclude that the formation of a ZR like many-body state similar to what is observed on the high $\mathrm{T}_{c}$ cuprates is indeed highly favorable in these systems with enhanced probability for the Cr compound.

\section{Discussion}

In summary, resonant soft $\mathrm{X}$-ray absorption data on $\mathrm{Cu} \mathrm{L}_{3,2^{-}}$and $\mathrm{O}$ $\mathrm{K}$-edges revealed the presence of the $\mathrm{ZR}$ singlet state in the symmetrically invariant $\mathrm{A}$-site-ordered cuprate compounds, $\mathrm{CaCu}_{3} \mathrm{Co}_{4} \mathrm{O}_{12}$ and $\mathrm{CaCu}_{3} \mathrm{Cr}_{4} \mathrm{O}_{12}$. Additionally, the data demonstrated the existence of a mixed valency for $\mathrm{Cu}$ modulated by the electronic state of the Bsite cation and reflected in the $\mathrm{d}^{9}, \mathrm{~d}^{9} \mathrm{~L}$, and the metastable $\mathrm{d}^{8}$ states of $\mathrm{Cu}$ d-electrons. First principles $\mathrm{GGA}+U$ calculations further validate the notion that within the A-site ordered perovskite family, the choice of B-site cation is an effective tool to manipulate the degree of $\mathrm{B}-\mathrm{Cu}-\mathrm{O}$ hybridization, adjusting the $\mathrm{Cu}$ valency towards the unusual $3+$ valence state in CCCoO and away from $3+$ closer to the stable $2+$ valency in CCCrO. Additionally, Wannier function calculations support this direct experimental observation that in the CCCrO compound the ZR many-body state is particularly favorable and controls the low-energy physics. These findings demonstrate that the charge and spin state of $\mathrm{Cu}$, fundamental to the intriguing physical properties the cuprates display, can be effectively altered by a careful choice of the B-site cation on isomorphic lattices that circumvent the chemical disorder and lattice modulation intrinsic to other doping methods.

\section{Methods}

Experimental details. Polycrystalline $\mathrm{CaCu}_{3} \mathrm{~B}_{4} \mathrm{O}_{12}(\mathrm{~B}=\mathrm{Cr}$ and $\mathrm{Co})$ samples were prepared under high-pressure and high-temperature (HPHT) conditions with a Walker-type multianvil module (Rockland Research Co.). For B $=\mathrm{Cr}$, stoichiometric amounts of $\mathrm{CaO}, \mathrm{CuO}$, and $\mathrm{CrO}_{2}$ powders were thoroughly mixed and then subjected to a HPHT treatment at $7 \mathrm{GPa}$ and $900^{\circ} \mathrm{C}$ for 30 minutes. For $\mathrm{B}=\mathrm{Co}$, a precursor containing $\mathrm{Ca}, \mathrm{Cu}$, and $\mathrm{Co}$ ions in the $1: 3: 4$ molar ratio obtained via a sol-gel route was mixed with $30 \mathrm{wt} \% \mathrm{KClO}_{4}$ acting as an oxidizing agent and then subjected to a $\mathrm{HPHT}$ treatment at $9 \mathrm{GPa}$ and $1000^{\circ} \mathrm{C}$ for 30 minutes. The resultant $\mathrm{KCl}$ was washed away with deionized water. Details about the sample assembly and procedures for the HPHT experiment can be found in a previous paper ${ }^{35}$. Phase purity of the above samples was examined with powder X-ray diffraction (XRD) at room temperature with a Philips Xpert diffractometer $(\mathrm{Cu} \mathrm{K} \alpha$ radiation). The $\mathrm{B}=\mathrm{Co}$ sample contains a small amount of cobalt and copper oxides. The cubic lattice parameter was calculated to be $\mathrm{a}=7.2385$ (5) and 7.1259(3) $\AA$ for $\mathrm{M}=\mathrm{Cr}$ and Co, respectively. Reitveld refinement for the two a-site ordered compounds is available in the Supplemental Figure S2.

LCO was synthesized under high oxygen pressure to the rhombohedral symmetry, details of which can be found elsewhere ${ }^{36}$. YBCO was grown with pulsed laser deposition, the details of which can also be found elsewhere ${ }^{37}$.

In order to elucidate the transformation of the $\mathrm{Cu}$ valency across these compounds, soft XAS measurements were carried out at the soft x-ray branch at the 4-ID-C beamline in the bulk-sensitive total fluorescence yield (TFY) mode and TEY mode in the Advanced Photon Source at Argonne National Laboratory. Measurements were taken on the $\mathrm{Cu}$ L-edge and O-K edge for all samples. All measurements were accompanied by a $\mathrm{CuO}$ standard, which allows precise alignment of the energy for oxidation state comparison. The Co and Cr L-edges were also obtained for the corresponding samples. All measurements shown were obtained in TFY mode; except, the Cr L edge data and all the Cr reference absorption data were taken in TEY mode.

Computational details. In the first-principles DFT calculations we have used the plane wave basis set and pseudo-potentials as implemented in the Vienna Ab-initio Simulation Package (VASP) ${ }^{38}$. The exchange-correlation function was chosen to be that of generalized gradient approximation (GGA) implemented following the parametrization of Perdew-Burke-Ernzerhof ${ }^{39}$. The electron-electron correlation beyond GGA was taken into account through improved treatment of GGA $+U$ calculation within the $+U$ implementation of Dudarev et al. ${ }^{40}$. For the plane wave based calculations, we used projector augmented wave (PAW $)^{41}$ potentials. The wave functions were expanded in the plane wave basis with a kinetic energy cutoff of $600 \mathrm{eV}$ and Brillouin zone summations were carried out with a $6 \times 6 \times 6 \mathrm{k}$-mesh. The $U$ value of $5 \mathrm{eV}$ on $\mathrm{Cu}$ site and $4 \mathrm{eV}$ on B site were used for GGA $+U$ calculations while the Hund's rule coupling $J$ was fixed to $0.8 \mathrm{eV}$. The obtained results were verified in terms of variation of $U$ parameter.

In order to estimate the positions of the $\mathrm{Cu}-\mathrm{d}, \mathrm{B}-\mathrm{d}$ and $\mathrm{O}-\mathrm{p}$ energy levels as well as the plots of the effective Wannier functions for B-d states, we used muffin-tin orbital (MTO) based N-th order MTO (NMTO) ${ }^{42}$-downfolding calculations. Starting from a full DFT calculations, NMTO-downfolding arrives at a few-orbital Hamiltonian by integrating out degrees which are not of interest. It does so by 
defining energy-selected, effective orbitals which serve as Wannier-like orbitals defining the few-orbital Hamiltonian in the downfolded representation. NMTO technique which is not yet available in its self-consistent form relies on the self-consistent potential parameters obtained out of linear muffin-tine orbital $(\text { LMTO })^{43}$ calculations. The results were cross-checked among the plane wave and LMTO calculations in terms of total energy differences, density of states and band structures.

1. Zaanen, J., Sawatzky, G. A. \& Allen, J. W. Band gaps and electronic structure of transition-metal compounds. Phys. Rev. Lett. 55, 418 (1985).

2. Plakida, Nikolay. High-Temperature Cuprate Superconductors. Berlin: SpringerVerlag, 2010.

3. Chakhalian, J. et al. Orbital Reconstruction and covalent bonding at an oxide interface. Science 318, 1114-1117 (2007).

4. Chakhalian, J. et al. Magnetism at the interface between ferromagnetic and superconducting oxides. Nat. Phys. 2, 244-8 (2006).

5. Karpinen, $\mathrm{M} \&$ Yamauchi, $\mathrm{H}$. The doping routes and distribution of holes in layered cuprates: a novel bond-valence approach. Phil. Mag. B 79, 2, 343-66 (1999).

6. Nücker, N., Fink, J., Fuggle, J. C., Durham, P. J. \& Temmerman, W. M. Evidence for holes on oxygen sites in the high $\mathrm{T}_{c}$ superconductors $\mathrm{La}_{2-x} \mathrm{Sr}_{x} \mathrm{CuO}_{4}$ and $\mathrm{YBa}_{2} \mathrm{Cu}_{3} \mathrm{O}_{7-\gamma}$. Phys. Rev. B 37, 10 (1988).

7. Nücker, N. et al. Site-specific and doping-dependent electronic structure of $\mathrm{YBa}_{2} \mathrm{Cu}_{3} \mathrm{O}_{x}$ probed by O1s and $\mathrm{Cu} 2 \mathrm{p} \mathrm{x}$-ray-absorption spectroscopy. Phys. Rev. B 51, 13 (1995).

8. Kuiper, P. et al. X-ray absorption study of the $\mathrm{O} 2 \mathrm{p}$ hole concentration dependence on $\mathrm{O}$ stoichiometry in $\mathrm{YBa}_{2} \mathrm{Cu}_{3} \mathrm{O}_{x}$. Phys. Rev. B 38, 10 (1988).

9. Zhang, F. C. \& Rice, T. M. Effective hamiltonian for the superconducting $\mathrm{Cu}$ oxides. Phys. Rev. B 37, 3759-3761 (1988).

10. Xiang, H. P., Liu, X. J., Meng, J. \& Wu, Z. J. Structural stability and magnetic coupling in $\mathrm{CaCu}_{3} \mathrm{Co}_{4} \mathrm{O}_{12}$ from first principles. J. Phys.: Condens Matter 21, 045501 (2009).

11. Shimakawa, Y. A-site-ordered perovskites with intriguing physical properties. Inorg. Chem 47, 8562-70 (2008).

12. Alippi, P. \& Fiorentini, V. Magnetism and unusual $\mathrm{Cu}$ valency in quadruple perovskites. Eur, Phys. J. B. 85, 82 (2012).

13. Tran, T. T., Takubo, K., Mizokawa, T., Kobayashi, W. \& Terasaki, I. Electronic structure of $\mathrm{CaCu}_{3} \mathrm{Ru}_{4} \mathrm{O}_{12}$ studied by x-ray photoemission spectroscopy. Phys. Rev. B 73, 193105 (2006).

14. Shiraki, H., Saito, T., Azuma, M. \& Shimakawa, Y. Metallic behavior n A-siteordered perovskites $\mathrm{ACu}_{3} \mathrm{~V}_{4} \mathrm{O}_{12}$ with $\mathrm{A}=\mathrm{Na}^{+}, \mathrm{Ca}^{2+}$, and $\mathrm{Y}^{3+}$. J. Phys. Soc. Jpn. 77, 6 (2008).

15. Mukherjee, S., Sarkar, S. \& Saha-Dasgupta, T. First Principles study of $\mathrm{CaCu}_{3} \mathrm{~B}_{4} \mathrm{O}_{12}(\mathrm{~B}=\mathrm{Co}$, Rh, Ir). J. Mater. Sci. 47, 7660-4 (2012).

16. Long, Y. W. et al. Temperature-induced A-B intersite charge transfer in a A-siteordered $\mathrm{LaCu}_{3} \mathrm{Fe}_{4} \mathrm{O}_{12}$ perovskite. Nature 458, 60 (2009).

17. McGuinness, C. et al. X-ray spectroscopic study of the electronic structure of the high-dielectric-constant material $\mathrm{CaCu}_{3} \mathrm{Ti}_{4} \mathrm{O}_{12}$. Phys. Rev. B 71, 19511 (2005).

18. Lin, Y., Chen, Y. B., Garret, T., Liu, S. W. \& Chen, C. L. Epitaxial growth of dielectric $\mathrm{CaCu}_{3} \mathrm{Ti}_{4} \mathrm{O}_{12}$ thin films on (001) $\mathrm{LaAlO}_{3}$ by pulsed laser deposition. Appl. Phys. Lett. 81, 631-3 (2002).

19. Yamada, I. et al. A perovskite containing quadrivalent iron as a chargedisproportionated ferrimagnet. Angew. Chem. Int. Ed 47, 7032-5 (2008)

20. Prodi, A. et al. Charge, orbital and spin ordering phenomena in the mixed valence manganite $\left(\mathrm{NaMn}^{3+}{ }_{3}\right)\left(\mathrm{Mn}^{3+}{ }_{2} \mathrm{Mn}^{4+}{ }_{2}\right) \mathrm{O}_{12}$. Nat. Mater. 3, 48-52 (2004).

21. Tanaka, S., Shimazui, N., Takatsu, H., Yonezawa, S. \& Maeno, Y. Heavy-Mass Behavior of Ordered Perovskites $\mathrm{ACu}_{3} \mathrm{Ru}_{4} \mathrm{O}_{12}(\mathrm{~A}=\mathrm{Na}, \mathrm{Ca}, \mathrm{La})$. J. Phys. Soc. Jpn. 78, 024706 (2009).

22. Hollmann, N. et al. Correlation effects in CaCu3Ru4O12 arXiv: $1211.2984 \mathrm{vl}$

23. Mizokawa, T. et al. Metallic versus insulating behavior in the A-site ordered perovskite oxides $\mathrm{ACu}_{3} \mathrm{Co}_{4} \mathrm{O}_{12}(\mathrm{~A}=\mathrm{Ca}$ and $\mathrm{Y})$ controlled by Mott and ZhangRice physics. Phys. Rev. B 80, 125105 (2009).

24. Subramanian, M. A., Marshall, W. J., Calvarese, T. G. \& Sleight, A. W. Valence degeneracy in $\mathrm{CaCu}_{3} \mathrm{Cr}_{4} \mathrm{O}_{12}$. J. Phys. and Chem. Solids 64, 1569-71 (2003).

25. Chen, C. T. et al. Electronic States in $\mathrm{La}_{2-x} \mathrm{Sr}_{x} \mathrm{CuO}_{4+\delta}$ probed by soft-x-ray absorption. Phys. Rev. Lett. 66, 1 (1991).

26. Grioni, M. et al. Studies of copper valence states with $\mathrm{Cu} \mathrm{L}_{3} \mathrm{x}$-ray absorption spectroscopy. Phys. Rev. B 39, 3 (1989).
27. Bianconi, A. et al. Linearly polarized $\mathrm{Cu} \mathrm{L}_{3}$-edge $\mathrm{x}$-ray-absorption near-edge structure of $\mathrm{Bi}_{2} \mathrm{CaSr}_{2} \mathrm{Cu}_{2} \mathrm{O}_{8}$. Phys. Rev. B 44, 18 (1991).

28. Mizokawa, T., Fujimori, A., Namatame, H., Takeda, Y. \& Takano, M. Electronic structure of tetragonal $\mathrm{LaCuO}_{3}$ studied by photoemission and $\mathrm{x}$-ray-absorption spectroscopy. Phys. Rev. B 57, 16 (1998).

29. Sarangi, R. et al. X-ray absorption edge spectroscopy and computational studies on $\mathrm{LCuO}_{2}$ species. J. Am. Chem. Soc. 128, 8286-96 (2006).

30. LeBoeuf, D. et al. Electron pockets in the Fermi surface of hole-doped high- $\mathrm{T}_{c}$ superconductors. Nature 450, 533-6 (2007).

31. Ando, Y., Komiya, S., Segawa, K., Ono, S. \& Kurita, Y. Electronic phase diagram of high- $\mathrm{T}_{c}$ cuprate superconductors from a mapping of the in-plane resistivity curvature. Phys. Rev. Lett. 93, 267001 (2004).

32. Sarma, D. D. et al. Electronic structure of high- $\mathrm{T}_{c}$ superconductors from soft-xray absorption. Phys. Rev. B 37, 16 (1988)

33. Debkov, Y. S. et al. Correlations in the electronic structure of half-metallic ferromagnetic $\mathrm{CrO}_{2}$ films: An x-ray absorption and resonant photoemission spectroscopy study. Phys. Rev. B 72, 060401(R) (2005).

34. Medling, S. et al. Evolution of magnetic oxygen states in Sr-doped $\mathrm{LaCoO}_{3}$. Phys. Rev. Lett. 109, 157204 (2012).

35. Cheng, J.-G., Zhou, J.-S. \& Goodenough, J. B. Evolution of ferromagnetism in orthorhombic perovskites $\mathrm{Sr}_{1-x} \mathrm{~Pb}_{x} \mathrm{RuO}_{3}$. Phys. Rev. B 81, 134412 (2010).

36. Zhou, J.-S., Archibald, W. \& Goodenough, J. B. Approach to Curie-Weiss paramagnetism in the metallic perovskites $\mathrm{La}_{1-x} \mathrm{Nd}_{x} \mathrm{CuO}_{3}$. Phys. Rev. B 61, 3196-3199 (2000).

37. Gray, B. A. et al. arXiv:1301.3736

38. Kresse, G. \& Furthmuller, J. Efficient iterative schemes for ab initio total-energy calculations using a plane-wave basis set. Phys. Rev. B 54, 11169 (1996).

39. P. Perdew, J., Burke, K. \& Ernzerhof, M. Generalized Gradient Approximation Made Simple. Phys. Rev. Lett. 77, 3865 (1996).

40. Dudarev, S. L., Botton, G. A., Savrasov, S. Y., Humphreys, C. J., \& Sutton, A. P. Electron-energy-loss spectra and the structural stability of nickel oxide: An LSDA + U study. Phys. Rev. B 57, 1505 (1998).

41. Blöchl, P. E. Projector augmented-wave method. Phys. Rev. B 50, 17953 (1994).

42. Andersen, O. K. \& Saha-Dasgupta, T. Muffin-tin orbitals of arbitrary order. Phys. Rev. B 62, R16219 (2000)

43. Andersen, O. K. \& Jepsen, O. Explicit, First-Principles Tight-Binding Theory Phys. Rev. Lett. 53, 2571 (1984)

\section{Acknowledgements}

JC is supported by DOD-ARO Grant No. 0402-17291. JSZ and JBG is supported by NSF Grant. No. DMR-1122603. TS-D would also like to thank, CSIR and DST, India for funding Work at the Advanced Photon Source, Argonne is supported by the U.S. Department of Energy, Office of Science under Grant No. DEAC02-06CH11357. Thanks to Dr. Bogdan Dabrowski for the $\mathrm{SrCoO}_{2.9}(\sim 4+)$ sample. JGC acknowledges the support from the Japan Society for the Promotion of Science (Grant No. 12F02023) and the Chinese Academy of Sciences.

\section{Author contributions}

D.M., J.W.F. and J.C. acquired the experimental data. S.M. and T.S.-D. did the theoretical calculations. J.G.C., J.S.Z. and J.B.G. grew the samples. S.M. and B.A.G. analyzed data and provided notes on the initial versions of the manuscript. D.M., T.S.-D. and J.C. wrote the final version of the manuscript.

\section{Additional information}

Supplementary information accompanies this paper at http://www.nature.com/ scientificreports

Competing financial interests: The authors declare no competing financial interests.

License: This work is licensed under a Creative Commons

Attribution-NonCommercial-NoDerivs 3.0 Unported License. To view a copy of this license, visit http://creativecommons.org/licenses/by-nc-nd/3.0/

How to cite this article: Meyers, D. et al. Zhang-Rice physics and anomalous copper states in A-site ordered perovskites. Sci. Rep. 3, 1834; DOI:10.1038/srep01834 (2013). 